\title{
Electronic Structure and Magnetic Properties of the Neptunyl Ion
}

\author{
J. C. Eisenstein and M. H. L. Pryce ${ }^{1}$
}

(December 29, 1964)

\begin{abstract}
The magnetic and spectroscopic properties of the neptunyl ion are reconsidered. The effect on the energy levels of covalency is discussed. New values for the parameters of our earlier theory are obtained, the analysis being based on more recent spectroscopic data and arguments concerning the relative widths and intensities of the optical absorption peaks. g-values and the temperature independent susceptibility are calculated and the results compared with experimental values. Experiments which need to be done to confirm various parts of the theory are suggested.
\end{abstract}

\section{Introduction}

Several years ago we $[1]^{2}$ published two papers (to be referred to here as I and II), on the properties of uranyl-like ions. Our interpretation of the experimental data was based on a specific model for these ions. We assumed that the uranium atom lies between, and equidistant from, the two oxygen atoms, the uranyl ion as a whole being predominantly covalently bonded. The ligand field, which is due to the bonding electrons, was assumed to be strong in comparison with the electrostatic field, which is due to neighboring ions or molecules.

Since 1956 important new spectroscopic measurements have been reported $[2,3]$. The new data do not require any revision in our model or in our general assumptions about the model. However, they do require rather large changes in the parameters of our theory such as the spinorbit coupling constant, the coulomb integrals and the strength of the ligand field.

In this paper and in a future paper on plutonyl a more complete discussion of the properties of the uranyl-like ions than appeared in our previous papers will be given. The object is not so much to present the revised values of the parameters as it is to discuss the numerous hypotheses which were made in attempting to understand the properties of these ions. Some of the things touched on in this paper are the most physically significant way of plotting an absorption spectrum, the interpretation of line widths, the possibility of fitting Gaussian curves to an unresolved spectrum, the excitation of vibrations, and the effects of covalent bond formation. The discussion of the general problems involved in understanding the behavior of uranyl-type ions is undoubtedly relevant to the interpretation of the properties of transition group compounds and other actinide compounds.

One of our most important contributions here is the revealing of the complexity of the uranyltype ions. For the neptunyl ion one can calculate exactly the positions of the energy levels for a variety of physically likely perturbations. The spectrum has been determined with sufficient accuracy so that one knows the experimental positions of the energy levels (except for uncertainties to be discussed). Consequently one can determine whether the likely perturbations are, or are not, adequate to account for the spectroscopic properties. A similar remark applies to the magnetic properties.

The available absorption spectra are those of neptunyl ions in water (or heavy water) and acid (commonly $\mathrm{HClO}_{4}$ ). The magnetic resonance data are for crystalline rubidium neptunyl

${ }^{1}$ Present address: Department of Physics, University of Southern California.

${ }^{2}$ Figures in brackets indicate the literature references at the end of this paper. 
nitrate diluted with the isomorphous uranyl salt. The susceptibility data are for crystalline sodium neptunyl acetate. It is therefore necessary to consider the neptunyl ion in different environments. That the environment can play a vital part is to be seen from I, where it is shown that the field $V_{6}^{6}$, due to the surrounding ions in the double nitrate, is responsible for the nonzero $g_{\perp}$, and contributes to reduce $g_{||}$from 4.0 to 3.4 . If the environment of the ion had a different symmetry, say a four-fold instead of a three-fold axis, the resonance behavior would be different; and if the ion were isolated, $g_{\perp}$ would be zero.

In our initial attempts to understand the solution absorption spectrum we paid no attention to the ionic environment (solvation sphere), but it now appears that this omission was unjustified. We are therefore led to the question: - what is the immediate environment?

In many crystals whose structure is known such as rubidium uranyl nitrate and sodium uranyl acetate the uranyl ion is surrounded by six oxygen atoms in an equatorial, hexagonal puckered ring. In sodium uranyl acetate, e.g., six oxygen atoms from three different acetate groups are almost coplanar with the uranium ion; in the (slightly) puckered oxygen ring there are two different $\mathrm{O}-\mathrm{O}$ distances [4]. Therefore it is reasonable to assume that in solution the uranyl ion is surrounded by six water molecules in a puckered ring. Such a system gives rise to a "crystalline" field with approximately three-fold symmetry. The axially symmetric components $V_{2}^{0}, V_{4}^{0}$, and $V_{6}^{0}$ of the "crystalline" field add to the ligand field of the ion itself, and modify the energy levels. The components $V_{4}^{3}, V_{6}^{3}$ and $V_{6}^{6}$ of the "crystalline" field mix the zero-order states which correspond to different axial quantum numbers, and lead to small changes in the energy levels. They also play a part in the selection rules for optical absorption.

There are also fluctuating deviations from these symmetric fields, which play a very important part in absorption by inducing electric dipole transitions.

In our earlier study of the spectrum we concentrated on the positions of the absorption bands and paid little attention to their strengths and widths. Since then a good deal of work has been published on the absorption spectra of compounds of transition elements, and the factors which determine strength and width are much more clearly understood. This work has furnished a number of important clues for the interpretation of the actinide spectra, most particularly for plutonyl.

\section{Energy Levels and Absorption Spectrum of Neptunyl}

The experimental absorption spectrum of $\mathrm{Np}(\mathrm{VI})$ is shown in figures 1 and 2 where Waggener's data are replotted as a function of wave number rather than wavelength. In addition we have used as ordinate the quantity $6900 \epsilon \lambda$ in place of the molar extinction coefficient. It is shown in

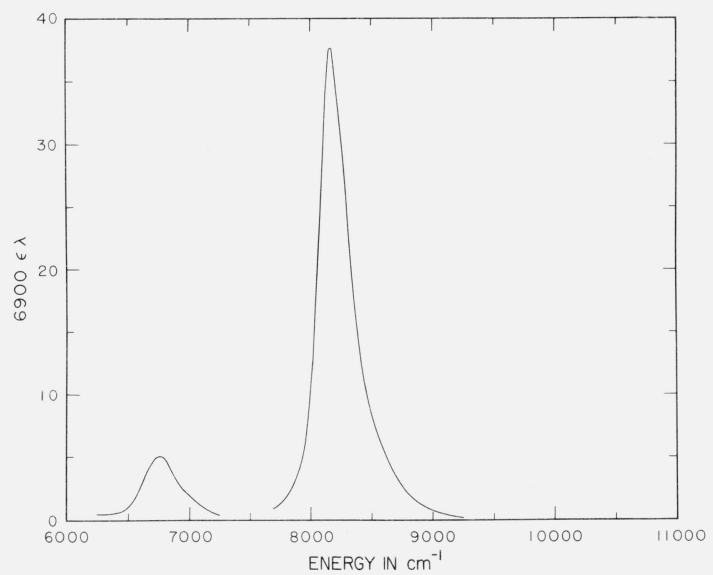

FIGURE 1. Absorption spectrum of the $\left(\mathrm{N}_{\mathrm{p}} \mathrm{O}_{2}\right)^{++}$ion from 6000 to $11000 \mathrm{~cm}^{-1}$.

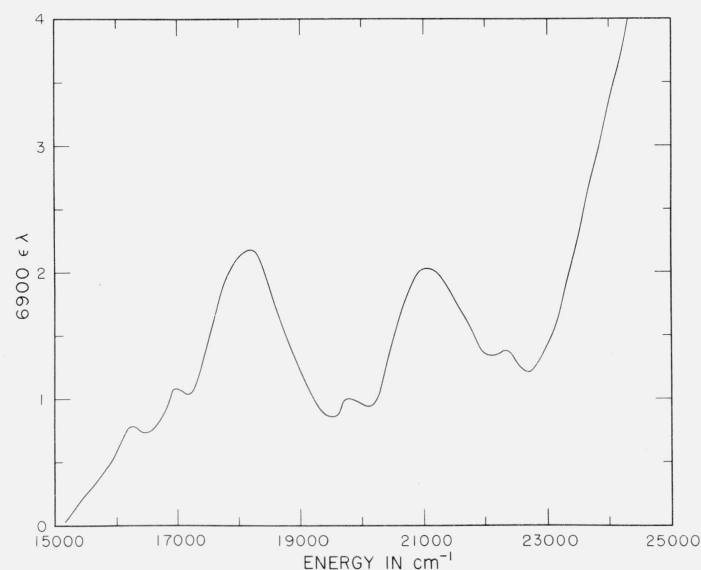

FiguRE 2. Absorption spectrum of the $\left(\mathrm{N}_{\mathrm{p}} \mathrm{O}_{2}\right)^{++}$ion from 15000 to $25000 \mathrm{~cm}^{-1}$. 
the appendix that $\epsilon \lambda$ is proportional to the transition probability. From the microscopic point of view, which we must adopt in analyzing the spectrum, $\epsilon \lambda$ is more physically meaningful than the extinction coefficient itself. The absorption peaks at 6752 and $8168 \mathrm{~cm}^{-1}$ were not known to exist prior to Waggener's and Hindman's work. The locations of these two peaks are the only new physical data on the neptunyl ion that have been published in the last ten years. The peaks at 18100 and $21100 \mathrm{~cm}^{-1}$ were known previously but have now been more precisely located.

We shall follow the conventions of papers I and II concerning the descriptions and labeling of the states. The zero order wave functions are taken to be the spherical harmonics $Y_{3}^{m}(\theta, \varphi)$ multiplied by appropriate spin functions, which we designate by + and - . When the spin-orbit interaction, the ligand field and the crystalline fields of three-fold and six-fold symmetry are taken into account one obtains the following matrix elements between the various states:

\begin{tabular}{|c|c|c|c|c|c|}
\hline & $\mid 3+>$ & $\mid-3+>$ & $\mid-2->$ & $\mid 1->$ & $\mid 0+>$ \\
\hline$<3+1$ & $\frac{3}{2} \zeta$ & $+V_{6}^{*}$ & 0 & 0 & $+\frac{1}{3} \sqrt{2 V_{3}^{\prime *}}$ \\
\hline$<-3+1$ & $+V_{6}$ & $-\frac{3}{2} \zeta$ & $\frac{1}{2} \sqrt{6} \zeta$ & 0 & $-\frac{1}{3} \sqrt{2} V_{3}^{\prime}$ \\
\hline$<-2-\|$ & 0 & $\frac{1}{2} \sqrt{6} \zeta$ & $X+\zeta$ & $V_{3}$ & 0 \\
\hline$<1-\mid$ & 0 & 0 & $V_{3}^{*}$ & $Y-\frac{1}{2} \zeta^{\prime \prime}$ & $\sqrt{3} \zeta^{\prime \prime \prime}$ \\
\hline \multirow[t]{2}{*}{$<0+1$} & $\frac{1}{3} \sqrt{2} V_{3}^{\prime}$ & $-\frac{1}{3} \sqrt{2} V_{3}^{\prime *}$ & 0 & $\sqrt{3} \zeta^{\prime \prime \prime}$ & $Z$ \\
\hline & $\mid 2->$ & $\mid 1+>$ & $\mid-2+>$ & $\mid-1->$ & \\
\hline$<2-1$ & $X-\zeta$ & $\frac{1}{2} \sqrt{10} \zeta^{\prime}$ & 0 & $-V_{3}^{*}$ & \\
\hline$<1+1$ & $\frac{1}{2} \sqrt{10} \zeta^{\prime}$ & $Y+\frac{1}{2} \zeta^{\prime \prime}$ & $V_{3}^{*}$ & 0 & \\
\hline$<-2+1$ & 0 & $V_{3}$ & $X-\zeta$ & $\frac{1}{2} \sqrt{10} \zeta^{\prime}$ & \\
\hline$<-1-1$ & $-V_{3}$ & 0 & $\frac{1}{2} \sqrt{10} \zeta^{\prime}$ & $Y+\frac{1}{2} \zeta^{\prime \prime}$ & \\
\hline
\end{tabular}

There is a matrix similar to the first one for the states $|-3->| 3-,>,|2+>|-1+.>$ and $\mid 0->$. It can be derived from the first one by observing that the crystalline potential is spinindependent; that $<2|V|-1>=-<1|V|-2>$; and that the matrix elements of spin-orbit interaction are identical.

$X, Y$, and $Z$ are the amounts the states with $m_{l}= \pm 2, \pm 1$ and 0 , respectively, are raised above the states with $m_{l}= \pm 3$ by the ligand field (including the axially symmetric parts of the crystalline field). $\quad V_{3}$ is the indicated matrix element of the part of the crystalline field which has three-fold symmetry about the $\mathrm{O}-\mathrm{U}-\mathrm{O}$ axis and $V_{6}$ is the matrix element of the part of the crystalline field which has six-fold symmetry. $\zeta$ is the spin-orbit coupling constant for a $5 f$ electron in the field of the $\mathrm{Np}^{7+}$ core.

The $5 f_{\sigma}$ (or $m_{l}=0$ ) orbitals are certainly used for bonding and there may also be some $\pi$-bonding which involves use of the $5 f_{\pi}$ (or $m_{l}= \pm 1$ ) orbitals. Consequently it is the antibonding orbitals containing the $5 f_{\sigma}$ and $5 f_{\pi}$ functions which are indicated schematically by $\left.|1+>| 0+,\right\rangle$, etc., in the matrices above. Since electrons in antibonding orbitals have a nonzero probability of being found on the oxygens, matrix elements of the spin-orbit interaction and the crystalline field must be appropriately reduced when these interactions connect an antibonding state with a nonbonding or antibonding state. It is for this reason that $\zeta^{\prime}, \zeta^{\prime \prime}$, and $\zeta^{\prime \prime \prime}$ appear instead of $\zeta$ in various places in the above matrices. $\zeta^{\prime}, \zeta^{\prime \prime}$, and $\zeta^{\prime \prime \prime}$ are reduced values of the spin-orbit coupling constant. For similar reasons the matrix element $\left\langle 3\left|V_{3}\right| 0\right\rangle$ should have a smaller value than one calculates when one assumes that the $5 f \mid 0>$ orbital is localized on the $\mathrm{Np}^{7+}$ core. 
As we shall see later, it is probable that the $m_{l}= \pm 3$ and $m_{l}= \pm 2$ orbitals are also contaminated by non-5f wave functions. Therefore the spin-orbit coupling constant $\zeta$ which appears in the preceding matrix elements may not have the value appropriate to an isolated neptunyl ion. Moreover, $\zeta$ need not have the same value for the states $\mid 3>$ and $|2\rangle$ but for simplicity we assume that it does. Evidently if $\zeta^{\prime}=\epsilon \zeta$ one should have $\zeta^{\prime \prime}=\epsilon^{2} \zeta$. In view of the probable contamination of the $m_{l}= \pm 3$ and $m_{l}= \pm 2$ orbitals it is possible that $\epsilon>1$. $\zeta^{\prime \prime \prime}$ should be appreciably smaller than the other values of $\zeta$ and not related to them in any simple way.

In I we assumed that the $5 f_{\sigma}$ state was so high that it had no important perturbing effect on the $m_{l}= \pm 3$ states through the $V_{3}$ interaction or on the $m_{l}= \pm 1$ states through the spin-orbit interaction. This assumption can no longer be justified, so the $\mid 0>$ states are retained in the present discussion.

In the absence of a magnetic field each state has two-fold (Kramers) degeneracy.

At the time we wrote our first paper we believed that the charge cloud due to the bonding electrons was the dominant factor in determining the sequence of energy levels. Since this charge cloud repels the odd electron from the ionic axis we expected the lowest doublet to be $\mid \pm 3 \mp>$. Measurement of the $g$-values has confirmed that this doublet is indeed the ground state. We expected the states with $m_{l}= \pm 2$ to lie higher and to be followed by the states with $m_{l}= \pm 1$. We expected the antibonding $m_{l}=0$ states to lie highest of all.

In 1955, the spectrum below $10000 \mathrm{~cm}^{-1}$ was still unexplored. Our original interpretation was based on the assumption that below the two levels corresponding to the observed peaks at 18100 and $21000 \mathrm{~cm}^{-1}$ there were only two others, namely those corresponding to the ground state $\mid \pm 3 \mp>$ and the other $\varphi$-state $\mid \pm 3 \pm>$. We therefore assigned the observed peaks to the states $\mid \pm 2 \mp>$ and $\mid \pm 2 \pm>$. The discovery of the two peaks at 6752 and $8168 \mathrm{~cm}^{-1}$ shows that the upper peaks must actually correspond to the states $\mid \pm 1 \mp>$ and $\mid \pm 1 \pm>$.

It follows from what has been said about the expected sequence of the levels that the prominent absorption peak at $8168 \mathrm{~cm}^{-1}$ must correspond to a transition from the ground state to one of the states $|3+>| 2-,>$ or $\mid 2+>$. (We omit the Kramers conjugate states for clarity.) The possibility that the $\mid 2->$ level occurs at $8168 \mathrm{~cm}^{-1}$ can be ruled out at once for, if true, the $\mid 2+>$ level would occur roughly $2 \zeta$ higher. However, there is no trace of an absorption peak between 8168 and $15600 \mathrm{~cm}^{1}$, and $\zeta$ can hardly be as large as $3700 \mathrm{~cm}^{-1}$.

On the other hand, the assignment of the $8168 \mathrm{~cm}^{-1}$ level to $\mid 2+>$ can be made with reasonable values of the parameters. If we ignore such refinements as $V_{3}, V_{3}^{\prime}, V_{6}$ and the distinction between $\zeta, \zeta^{\prime}$, etc., we have four parameters at our disposal $(X, Y, Z$, and $\zeta)$, and four observations to fit (the levels at $6752,8168,18100$, and $21100 \mathrm{~cm}^{-1}$ ). Therefore we can probably find values of the parameters such that the calculated energy levels agree with the data. In fact, if we set $X=1608 \mathrm{~cm}^{-1}, Y=15776 \mathrm{~cm}^{-1}, Z=39995 \mathrm{~cm}^{-1}, \zeta=\zeta^{\prime}=\zeta^{\prime \prime}=\zeta^{\prime \prime \prime}=1984 \mathrm{~cm}^{-1}, V_{3}=V_{3}^{\prime}=V_{6}=0$ we find the following energy level positions:

\begin{tabular}{lr}
\hline \hline & $c m^{-1}$ \\
$E(3-)$ & 0 \\
$E(2-)$ & 2846 \\
$E(3+)$ & 6752 \\
$E(2+)$ & 8168 \\
$E(1-)$ & 18100 \\
$E(1+)$ & 21000 \\
$E(0+)$ & 40455
\end{tabular}

These calculated levels are in exact agreement with the experimental absorption spectrum. Of course, no critical confirmation of the theory is achieved since there are enough adjustable parameters to make this agreement possible. However one also finds that the calculated $g$-values agree with the measured values [5] on rubidium neptunyl nitrate and the calculated temperature-independent susceptibility agrees with the value measured [6] for sodium neptunyl acetate, if suitable assumptions concerning $V_{6}$ and $V_{3}$ are made. 
Despite this perfect accord between theory and experiment, and despite some uncertainties (to be discussed below) connected with the remaining alternative possibility, we prefer to associate the $\mid 3+>$ state with the level at $8168 \mathrm{~cm}^{-1}$.

The arguments which have led us to adopt this view come mainly from our analysis of the plutonyl spectrum, and will be reviewed more critically in a subsequent paper which will be devoted to $\mathrm{PuO}_{2}^{++}$and $\mathrm{NpO}_{2}^{+}$. Here it will suffice to outline their nature.

The spectrum of $\mathrm{PuO}_{2}^{++}$is remarkable for the presence of a very strong, very narrow absorption band at $12050 \mathrm{~cm}^{-1}$. Its extreme sharpness suggests most strongly that the electronic charge distribution in the upper state is almost identical with that in the ground state, so that fluctuating electric fields have negligible effect on the energy separation of the two levels. To a first approximation the ground state of $\mathrm{PuO}_{2}^{++}$(with two 5 -electrons) is the degenerate doublet $\mid 3-, 2->$ and $\mid-3+,-2+>$. We are convinced that the upper level at $12050 \mathrm{~cm}^{-1}$ is the doublet approximating to $\mid-3-, 2->$ and $\mid 3+,-2+>$. The electronic transition corresponds to a change in the orbital quantum number $m_{l}$ of one of the electrons from -3 to 3 , or conversely - without change of spin. The charge distribution is not changed by this transition, and the absorption peak is therefore narrow. A further step in the argument concerns the strength of the absorption. If one knew the matrix elements which determine the absorption of light by the one-electron system $\mathrm{NpO}_{2}^{++}$, and the compositions of the states of the two-electron system $\mathrm{PuO}_{2}^{++}$(or $\mathrm{NpO}_{2}^{+}$), and if one assumed the inner fields and environment to be the same for the two ions, one could estimate the strengths of the absorption bands of $\mathrm{PuO}_{2}^{++}\left(\right.$or $\mathrm{NpO}_{2}^{+}$). Now, it is remarkable that the strength of the dominant line in the plutonyl spectrum agrees very closely with that of the dominant line at $8168 \mathrm{~cm}^{-1}$ in the neptunyl spectrum. We are therefore led to conclude that the transition involved is the same in the two cases, i.e., from $\mid \pm 3 \mp>$ to $\mid \mp 3 \mp>$. It is on this basis that we have assigned the $8168 \mathrm{~cm}^{-1}$ level to $\mid \pm 3 \pm>$.

If we set $X=-1716 \mathrm{~cm}^{-1}, Y=14558 \mathrm{~cm}^{-1}, Z=131102 \mathrm{~cm}^{-1}, \zeta=\zeta^{\prime}=\zeta^{\prime \prime}=\zeta^{\prime \prime \prime}=2248 \mathrm{~cm}^{-1}$, and $V_{3}=V_{3}^{\prime}=V_{6}=0$ we obtain the following energy levels:

\begin{tabular}{l|r}
\hline \hline & \\
$E(3-)$ & $\mathrm{cm}^{-1}$ \\
$E(2-)$ & 0 \\
$E(2+)$ & 208 \\
$E(3+)$ & 6750 \\
$E(1-)$ & 8167 \\
$E(1+)$ & 18100 \\
$E(0+)$ & 21100 \\
\hline
\end{tabular}

Again there is precise agreement with experiment.

Two objections to our proposed assignments follow from the figures just quoted. In the first place, $X$ is negative; that is, the $m_{l}= \pm 2$ orbitals are below the $m_{l}= \pm 3$ orbitals whereas we had expected the reverse to be the case. To account for this negative value of $X$ we must assume that the surrounding ions or molecules give rise to a field with axial symmetry which repels the odd electron from the equatorial plane. The existence of such a field follows from our model of the solvation sphere, with six water molecules in a puckered ring.

The second objection is to the low position $\left(200 \mathrm{~cm}^{-1}\right)$ of the $\mid 2->$ state. If it were really as low as this, it would be appreciably populated at room temperature, and would give rise to satellite absorption peaks $200 \mathrm{~cm}^{-1}$ to the red of the main peaks. Although such peaks would not be resolved, their presence would be detectable, but the spectrum shows no sign of them. In addition, if the $\mid 2->$ state were as low as $200 \mathrm{~cm}^{-1}$ the calculated temperature-independent susceptibility would be much larger than the measured value. However, when such refinements as $V_{6}$, $\zeta^{\prime}$, etc., are considered, the calculated position of the $\mid 2->$ level relative to $|3-\rangle$ is raised to $500-1000 \mathrm{~cm}^{-1}$. Therefore the difficulties associated with the position of the $\mid 2->$ level may be phantasmal. 
Possibly the ligand and crystalline fields are not the same in a solution as they are in a crystalline solid. However, such an ad hoc hypothesis to account for the depression of the $m_{l}= \pm 2$ states relative to the $m_{l}= \pm 3$ states in solution does not appear very satisfactory. It is also possible that the very difficult susceptibility measurements are in error. It would be interesting to have these measurements extended to a range of neptunyl compounds. Better still, it would be valuable to study the optical absorption, magnetic resonance and susceptibility of the same compound.

The two hypotheses described above, both of which are adequate for interpreting the positions of the energy levels, differ in the value of the spin-orbit coupling constant required. One might think that it would be possible to decide between the two hypotheses if the value of $\zeta$ for some other hexavalent neptunium compound were known. Recently magnetic and spectroscopic data on $\mathrm{NpF}_{6}$ have become available. Unfortunately, the unambiguous determination of the value of $\zeta$ from these data has proved difficult.

Our analysis [7] of the data on $\mathrm{NpF}_{6}$ led to two possible interpretations, neither of which was wholly satisfactory. One, which accounted for the magnetic resonance and susceptibility data, and gave energy levels at the positions of the two known absorption bands in the near infrared, required $\zeta=1941 \mathrm{~cm}^{-1}$. Its defects were that the ratios of the ligand field parameters which entered into the theory were not very plausible on the basis of our model of the molecular structure; and that it predisted an infrared absorption band around $3000 \mathrm{~cm}^{-1}$, which has not been found experimentally. The other, requiring $\zeta=2405 \mathrm{~cm}^{-1}$, accounted satisfactorily for the positions of all the absorption bands, including some in the ultraviolet whose ascription to $5 f-5 f$ transitions is by no means certain, but it led to a value for the temperature-independent part of the susceptibility which is much lower than is observed.

It is tempting to think that the agreement between the numbers $1941 \mathrm{~cm}^{-1}$ for $\mathrm{NpF}_{6}$ and 1985 $\mathrm{cm}^{-1}$ for $\mathrm{NpO}_{2}^{++}$indicates that the "correct" value for the spin-orbit coupling constant is close to these figures. Other arguments for accepting the value $1985 \mathrm{~cm}^{-1}$ are given above. Nevertheless, we think that the spin-orbit coupling constant for the neptunyl ion probably has a value near $2200 \mathrm{~cm}^{-1}$, and the higher value of $\zeta$ for $\mathrm{NpF}_{6}$ is also probably the correct one.

\section{General Considerations Concerning the Interpretation of the Spectrum}

The principal features of the absorption spectrum are apparent on casual inspection of figures 1 and 2. There are four peaks below $22000 \mathrm{~cm}^{-1}$ which we ascribe to electronic transitions within the $5 f$ shell. The steep rise of the absorption curve above $23000 \mathrm{~cm}^{-1}$ is probably due to an allowed $5 f$ to $6 d$ transition. The four subsidiary peaks are probably part of the vibrational fine structure.

As we have already indicated, the interpretation of the spectrum is not free from ambiguity. In addition to the difficulty connected with assigning peaks to particular electronic levels there is uncertainty in the interpretation because of the widths of the peaks and (in some cases) their vibrational fine structures.

We must remember that we are not dealing with an ion in a fixed environment, but with an ion whose environment adapts itself to the ion. Energies are therefore not those of an isolated ion, but those of the whole system. It would be too ambitious to try to deal with this system in its full generality; it seems wiser to ask ourselves what would be the energy levels of the ion in the environment which it has in its ground state. Even this question is imprecise, for that environment is not a fixed one. We can restore precision to the question we wish to answer by postulating some static mean environment. When we are calculating magnetic properties in the ground state, the energies in the mean environment are the ones which are relevant.

There is a distinction between the energies so defined and the energies actually observed (as frequencies) in an absorption spectrum, for the equilibrium configuration of the environment will be altered for the excited electronic states of the ion. The ionic energy as we propose to study it is higher than the energy of the system with the ion in its excited electronic state and the environ- 
ment in equilibrium with that state. The result is that the electronic transition is accompanied by the excitation of vibrational motion in the environment, and the transition is not sharp. This excitation is responsible, in part, for the line width.

Furthermore we are dealing with a complex ion which is itself capable of internal vibration. In excited states the equilibrium $\mathrm{Np}-\mathrm{O}$ bond distance is not the same as in the ground state, and vibrations, notably of the symmetrical stretching mode, can be excited in an electronic transition. Therefore vibrational fine structure is observed in the absorption spectrum.

It is also important to remember that we are dealing with optical transitions between levels of the same electronic parity $\left(5 f_{u} \rightarrow 5 f_{u}\right)$. The transitions are therefore forbidden. Quantitative analysis of their intensity shows that the two dominant mechanisms available to parity-forbidden transitions in atomic spectroscopy, namely magnetic dipole and electric quadrupole couplings to the electromagnetic field, are much too weak to account for the observed absorption bands. The only mechanism capable of giving the observed absorption is electric dipole coupling induced by the fluctuating field of the environment. This coupling causes departures from the perfect odd parity of the electronic states, and thereby allows nonvanishing matrix elements of electric dipole moment.

In the fluctuating fields arising from distortions and motions of the solvation sphere and from vibrations of the ion, nearly all the selection rules of atomic and molecular spectroscopy break down. One, however, remains in modified form, namely the rule that in an optical transition there is no change of spin quantum number. Since the "crystalline" fields and the electric dipole moment are spin-independent, no perturbation by these fields can give transition matrix elements between states of different spin.

It will be seen in this and subsequent papers that although changes in $m_{l}$ from 0 to \pm 6 are encountered, $\Delta m_{s}$ is zero in all except some very weak bands. (This statement needs qualification when $m_{s}$ is not a good quantum number, and we shall discuss it more precisely in a later paper.) Indeed we shall see that the strongest bands correspond to $\Delta m_{l}= \pm 6$.

All of the absorption peaks are rather broad and asymmetrical. Consequently it is not clear what energies should be used as the positions of the electronic levels. If we consider only first order couplings with the environment, and if an absorption peak is not too broad, then it can be shown that the centroid ${ }^{3}$ of the peak corresponds to the sum of the ionic energy, as we have defined it, and a positive correction which is connected with the transfer of energy to the vibrations of odd parity which induce the electric moment in these otherwise parity-forbidden transitions. When the fluctuations which give rise to the dipole moment are slow, as we believe to be the case here, this correction is negligible. In any case, the first order perturbations will not lead to any asymmetry of the absorption peaks.

If the width and asymmetry of a peak are due to random depression of the ground state by second order coupling with the nearby $\mid 2->$ state then the energy shift is given by $\left(V^{2}+W^{2}\right) / \Delta E$, where $V$ and $W$ are the real and imaginary parts of the appropriate matrix element, and $\Delta E$ is the energy gap between the two states. It is reasonable to assume that $V$ and $W$ can be represented by Gaussian distributions with equal amplitudes and widths. The line shape corresponding to these assumptions can easily be calculated. The absorption jumps from zero to a finite value at the threshold and falls off exponentially thereafter. In order to account for the observed line shapes it is necessary to make additional assumptions; for example, that second order perturbations also move the upper levels about. We will not push this possible interpretation any further.

An alternative and more probable explanation of the marked asymmetry of the absorption peaks is that it is due to unresolved vibrational fine structure. We can get a rough idea of the positions, amplitudes and widths of the peaks by fitting a sum of Gaussian functions to the observed absorption curve. This fitting lacks any theoretical justification so it should not be taken too seriously. In the following discussion the amplitudes are expressed in units of $6900 \epsilon \lambda$ where $\epsilon$ is the molar extinction coefficient and $\lambda$ is the wavelength in $\mathrm{cm}$. 
After making an allowance of 0.39 units for the background one finds that the peak whose maximum is at $6752 \mathrm{~cm}^{-1}$ can be fitted by a Gaussian function whose amplitude is 4.70 and whose full width at half maximum is $292 \mathrm{~cm}^{-1}$. A small peak is left over, centered at about $7039 \mathrm{~cm}^{-1}$, with an amplitude of 0.90 and width $210 \mathrm{~cm}^{-1}$. These two peaks together fit 42 points read off Waggener's curve with a maximum deviation of 0.10 and a root mean square deviation of 0.047 .

The large absorption peak at $8168 \mathrm{~cm}^{-1}$ may have as many as four subsidiary peaks on the high frequency side and two on the low frequency side.

According to our analysis (which, it must be admitted, is not unique) the separation of the unresolved peaks is about $200 \mathrm{~cm}^{-1}$. The bending frequency in the ground state of the uranyl ion is known to be about $215 \mathrm{~cm}^{-1}$. Therefore it is possible to assign all the subsidiary peaks below $10000 \mathrm{~cm}^{-1}$ to vibrational fine structure. The peaks on the high frequency side of the main peaks may be due to the electronic transitions accompanied by excitation of the bending mode. It seems reasonable that the bending vibrations rather than the stretching vibrations should be involved here since, in both ground and excited electronic states, the charge distribution is near the equatorial plane.

One puzzling feature is that the $8168 \mathrm{~cm}^{-1}$ peak is almost as wide $\left(233 \mathrm{~cm}^{-1}\right)$ as the $6752 \mathrm{~cm}^{-1}$ peak. In first approximation it corresponds to a transition from $m_{l}= \pm 3$ to $m_{l}=\mp 3$, involving no change of charge distribution, and therefore should be very narrow. Even when allowance is made for the contamination of the ground state by $\mid 2+>$, the change in charge distribution is still considerably less (by a factor of at least 2) than for the $6752 \mathrm{~cm}^{-1}$ line. It may be that the nearby $\mid 2->$ state perturbs the ground state. Fluctuating fields which couple the two states might cause them to spread in energy over a range sufficient to account for the $233 \mathrm{~cm}^{-1}$ width. In plutonyl, where the lowest excited level is at $2500 \mathrm{~cm}^{-1}$, the width of the $12050 \mathrm{~cm}^{-1}$ peak, which should be closely comparable with the $8168 \mathrm{~cm}^{-1}$ peak in neptunyl, is less than $50 \mathrm{~cm}^{-1}$. The fact that the $6752 \mathrm{~cm}^{-1}$ peak is (slightly) wider than the $8168 \mathrm{~cm}^{-1}$ peak is a point in favor of the assignment of the upper levels to $\mid 2+>$ and $\mid 3+>$ respectively.

It is more difficult to analyze the complex structure of the absorption spectrum above 15000 $\mathrm{cm}^{-1}$. However, the set of Gaussian curves whose descriptions follow yields a representation of the absorption curve which accords very closely with the data.

\begin{tabular}{c|r|r|r}
\hline \hline Center & Amplitude & $\begin{array}{c}\text { Width at half } \\
\text { maximum }\end{array}$ & Strength \\
\hline$c^{-1}$ & & $\mathrm{~cm}^{-1}$ & \\
15670 & 0.300 & 588 & 0.97 \\
16245 & .690 & 588 & 2.23 \\
16850 & .620 & 588 & 2.00 \\
18100 & 2.180 & 1540 & 18.44 \\
18590 & 0.077 & 588 & 0.25 \\
19150 & .410 & 588 & 1.32 \\
19760 & .550 & 588 & 1.78 \\
21100 & 2.000 & 5860 & 1.24 \\
22330 & 0.352 & 3241 & $\ldots \ldots \ldots$ \\
25210 & 4.933 & & 14.2 \\
\hline
\end{tabular}

The sum of these curves has a maximum deviation of 0.07 from 75 points read off $\mathrm{W}$ aggener's curve between 15000 and $24400 \mathrm{~cm}^{-1}$. The root mean square deviation is 0.024 . It is rather tedious to fit Gaussian curves to the experimental spectrum so no serious effort was made to find the set which minimizes the root mean square deviation. Such an effort is likely to be unrewarding in view of the unavoidable errors in plotting the experimental curve, and in reading points off it.

Although one can argue about the details, the following general points seem to be well established. (1) The two principal absorption peaks at 18100 and $21100 \mathrm{~cm}^{-1}$ have nearly the same widths $\left(1600 \mathrm{~cm}^{-1}\right)$ and strengths (18.3). (2) There are three subsidiary peaks at 15670,16245 , 
and $16850 \mathrm{~cm}^{-1}$. (3) There are two subsidiary peaks at 19150 and $19760 \mathrm{~cm}^{-1}$ and possibly a third at $18590 \mathrm{~cm}^{-1}$. (4) There is a subsidiary peak at $22330 \mathrm{~cm}^{-1}$. (5) All the subsidiary peaks have nearly the same width of $590 \mathrm{~cm}^{-1}$. They are very much narrower than the principal peaks.

The similarity of the two principal peaks is strong evidence in favor of our hypothesis that they are both due to transitions to an orbital state with $\left|m_{l}\right|=1$. The width of these peaks is possibly associated with nonequilibrium configurations of the ion and its environment.

The subsidiary peaks listed under (2) and (3) above are quite possibly not associated with the principal peaks. In the first place, they are definitely narrower than the latter. Secondly, they seem to form two similar groups; one group occurs on the low frequency side of each principal peak. Consequently they probably cannot be ascribed to transitions in which vibrational modes are excited. The strengths of the subsidiary peaks are too great for the peaks to be ascribed to transitions from the vibrationally excited ground electronic state.

We are inclined to attribute the subsidiary peaks below $20000 \mathrm{~cm}^{-1}$ to transitions in which, in the final electronic state, the environment has accommodated itself to the altered charge distribution of the ion. That is, the energies $15670 \mathrm{~cm}^{-1}, 16245 \mathrm{~cm}^{-1}$, etc. correspond to states in which the environment is distorted away from its ground state configuration toward that appropriate to the $\mid 1->$ and $\mid 1+>$ electronic states.

The energy differences of the successive peaks listed under (2) and (3) are all approximately $600 \mathrm{~cm}^{-1}$. This difference possibly represents the energy of the symmetric stretching vibration in the excited state. That the stretching vibrations of the neptunyl ion should be strongly excited is plausible, for the electron in the $m_{l}= \pm 1$ upper state is much closer to the bonding electrons than when in the ground state, so that the equilibrium bond distance must be considerably changed. On the other hand, $600 \mathrm{~cm}^{-1}$ is a rather low energy for the stretching vibration. It is a moot question whether the energy of this vibration can change from about $860 \mathrm{~cm}^{-1}$ in the ground state to 600 $\mathrm{cm}^{-1}$ in an excited state.

The small peak at $22330 \mathrm{~cm}^{-1}$ is probably part of the vibrational fine structure of the principal peak at $21100 \mathrm{~cm}^{-1}$. If so, its position $(21100+2 \times 615)$ indicates that the energy of the stretching vibration really is about $600 \mathrm{~cm}^{-1}$. Because of the strongly rising absorption above $22600 \mathrm{~cm}^{-1}$ it is impossible accurately to determine the width of the $22330 \mathrm{~cm}^{-1}$ peak. It may very well be greater than the $588 \mathrm{~cm}^{-1}$ quoted above.

\section{Values for the Empirical Parameters of the Theory}

In comparing the theoretical and experimental values of the coefficients in the spin Hamiltonian, and for the purpose of extending the theory to other uranyl-type ions, it is important to know the extent to which the observed neptunyl absorption peaks fix the values of the parameters. We have already introduced ten of these parameters: $X, Y, Z, \zeta, \zeta^{\prime}, \zeta^{\prime \prime}, \zeta^{\prime \prime \prime}, V_{3}, V_{3}^{\prime}$, and $V_{6}$. The locations of the four absorption peaks provide only four relations among them. Roughly speaking they determine $X, Y$, and $\zeta$ fairly closely, and $Z$ to within 50 percent. Varying the remaining parameters $\left(\zeta, \zeta^{\prime}, \zeta^{\prime \prime}, \zeta^{\prime \prime \prime}\right.$ remaining roughly equal) causes only slight changes in $X, Y$, and $\zeta$. $V_{6}$ has a strong effect on $g_{\perp}$, and can be estimated from that. $g_{\|}$is sensitive to $V_{6}$ and $V_{3}^{\prime}$, and is also very sensitive to " $l$-reduction," i.e., a loss of $5 f$-character in the $|3-\rangle$ wave function due to partial overlap with the orbitals on the six equatorial oxygen atoms. This $l$-reduction is very similar to the mechanism which reduces $\zeta$ to $\zeta^{\prime}$ etc., but applies to the $\mid \pm 3>$ states. The susceptibility is extremely sensitive to the position of the $\mid 2->$ level.

Clearly we cannot determine all the parameters of the theory from the experimental data. Rather, we must attempt to show that reasonable values for the parameters, suggested by the nature of the model, are capable of explaining the salient features of the observations. Our procedure will be to assign values to the crystal field matrix elements, and then show how the remaining parameters are related. 
The simplest case to treat is that for which $V_{3}=V_{3}^{\prime}=V_{6}=0$. In this case the secular equations can be factored so that the most complicated equation one has to solve is quadratic in the energy. The experimental positions of the $\mid 2+>$ and $\mid 3+>$ levels determine $\zeta$ and $X$ uniquely. One finds $\zeta=2248 \mathrm{~cm}^{-1}$ and $X=-1716 \mathrm{~cm}^{-1}$.

Next, using the information that $\mid 1+>$ is at $21100 \mathrm{~cm}^{-1}$, we can determine $Y$. It will be seen that the relevant energy matrix contains $\zeta^{\prime}$ and $\zeta^{\prime \prime}$ as well as $\zeta$. We assume that $\zeta^{\prime}=\epsilon \zeta$ and $\zeta^{\prime \prime}=\epsilon^{2} \zeta$ where $\epsilon$ is a parameter probably less than but near 1 . One easily finds that

$$
Y=16305-1747.3 \epsilon^{2}
$$

The energy of the $\mid 2->$ state is the other eigenvalue of the matrix and can be determined when $Y$ is known. One finds that $E(2-)=831-623.3 \epsilon^{2}$.

Having found $Y$ we can now use the position of the $\mid 1->$ level, $18100 \mathrm{~cm}^{-1}$, to determine $Z$. $\zeta^{\prime \prime \prime}$ appears in this calculation as well as $\epsilon$. The calculation also determines the energy of the $\mid 0 \pm>$ level. One finds that

$$
\begin{gathered}
Z=13305+\frac{\zeta^{\prime \prime \prime 2}}{1000-957.1 \epsilon^{2}} \\
E(0 \pm)=21100-2871.3 \epsilon^{2}+\frac{\zeta^{\prime \prime \prime 2}}{1000-957.1 \epsilon^{2}} .
\end{gathered}
$$

All of these formulas are exact; that is, they do not result from perturbation calculations. Results for various values of $\epsilon^{2}$ are given in the following table:

\begin{tabular}{r|c|c|c|c}
\hline \hline $\boldsymbol{\epsilon}^{2}$ & $Y\left(\mathrm{~cm}^{-1}\right)$ & $Z\left(\mathrm{~cm}^{-1}\right)$ & $E(2-) \mathrm{cm}^{-1}$ & $E(0 \pm) \mathrm{cm}^{-1}$ \\
\hline & & & & \\
1.0 & 14558 & $13305+\zeta^{\prime \prime \prime 2} / 42.9$ & 208 & $18229+\zeta^{\prime \prime \prime 2} / 42.9$ \\
0.9 & 14732 & $13305+\zeta^{\prime \prime \prime 2} / 138.6$ & 270 & $18516+\zeta^{\prime \prime \prime 2} / 138.6$ \\
.8 & 14907 & $13305+\zeta^{\prime \prime \prime 2} / 234.3$ & 332 & $18803+\zeta^{\prime \prime \prime 2} / 234.3$ \\
.7 & 15082 & $13305+\zeta^{\prime \prime \prime 2} / 330.0$ & 395 & $19090+\zeta^{\prime \prime \prime 2} / 330.0$ \\
\hline
\end{tabular}

We obtain a very rough value for $Z$ by observing that $\zeta^{\prime \prime \prime}$ is less than $\zeta$ but probably more than $\frac{1}{2} \zeta$. It follows that $Z$ lies between about $131000 \mathrm{~cm}^{-1}$ (for $\epsilon^{2}=1, \zeta^{\prime \prime}=\zeta$ ) and $17100 \mathrm{~cm}^{-1}$ (for $\epsilon^{2}=0.7, \zeta^{\prime \prime}=\frac{1}{2} \zeta$ ). This determination of $Z$ is particularly sensitive to the separation between the $\mid 1+>$ and $\mid 1->$ levels. Since this separation cannot be accurately determined from the spectrum, not much can be deduced concerning $Z$.

On the basis of our model we expected $Z$ to be greater than $Y$ (perhaps $Z>25000 \mathrm{~cm}^{-1}$ ), and perhaps less than $50000 \mathrm{~cm}^{-1}$. No conflict occurs.

Essentially what we learn from the preceding calculation is that our interpretation of the 18100 and $21100 \mathrm{~cm}^{-1}$ peaks as the doublet $\mid 1->$ and $\mid 1+>$ is consistent with the value of $\zeta$ deduced from the infrared peaks, provided $\epsilon^{2}$ and $\zeta^{\prime \prime \prime}$ are suitably chosen. A more significant way of presenting the results of these calculations is embodied in the following equation:

$$
\epsilon^{2}=1.045-\frac{\zeta^{\prime \prime \prime 2}}{957(Z-13305)} \pm 0.070
$$

The indicated uncertainty is the effect of the $\pm 200 \mathrm{~cm}^{-1}$ possible error in the separation between the peaks at 18100 and $21100 \mathrm{~cm}^{-1}$. Since $\epsilon^{2}$ is probably near 1 , we see that some limitation is imposed on our assumptions concerning the values of $Z$ and $\zeta^{\prime \prime \prime}$, but that the uncertainties in the observational values are such that these constraints are practically ineffective. 
The next simplest case to treat is that in which $V_{3}^{\prime}=V_{3}=0$ and $V_{6}$ differs from zero. We shall take $V_{6}$ to be $1500 \mathrm{~cm}^{-1}$, since this order of magnitude is suggested by resonance data. Again the values of $\zeta$ and $X$ are uniquely determined by the positions of the $\mid 2+>$ and $\mid 3+>$ levels. One finds $\zeta=2071 \mathrm{~cm}^{-1}, X=-599 \mathrm{~cm}^{-1}$. With this choice of $\zeta$ and $X, E(2+)$ is $6752 \mathrm{~cm}^{-1}$ and $E(3+)$ is $8168 \mathrm{~cm}^{-1}$. One can then determine $Y, Z, E(2-)$ and $E(0 \pm)$ as before. One finds the results given in the following table.

\begin{tabular}{c|c|c|c|c}
\hline$\epsilon^{2}$ & $Y\left(\mathrm{~cm}^{-1}\right)$ & $Z\left(\mathrm{~cm}^{-1}\right)$ & $E(2-)\left(\mathrm{cm}^{-1}\right)$ & $E(0 \pm)\left(\mathrm{cm}^{-1}\right)$ \\
\hline & & & & 1257 \\
1.0 & 15026 & $13617+\zeta^{\prime \prime \prime 2} / 124.35$ & 1313 & $18473+\zeta^{\prime \prime \prime 2} / 124.35$ \\
0.9 & 15185 & $13617+\zeta^{\prime \prime \prime 2} / 211.91$ & 1368 & $18736+\zeta^{\prime \prime \prime 2} / 211.91$ \\
0.8 & 15344 & $13617+\zeta^{\prime \prime \prime 2} / 299.48$ & 1424 & $1998+\zeta^{\prime \prime \prime 2} / 299.48$ \\
0.7 & 15503 & $13617+\zeta^{\prime \prime \prime 2} / 387.05$ & & $19261+\zeta^{\prime \prime \prime 2} / 387.05$ \\
\hline
\end{tabular}

The uncertainty concerning $Z$ can be expressed as before by an equation:

$$
\epsilon^{2}=1.142-\frac{\zeta^{\prime \prime \prime 2}}{8.75 .65(Z-13617)} \pm 0.076 .
$$

Finally we shall obtain values of $\zeta, X, Y$, and $Z$ for the case $V_{3}^{\prime}=\frac{1}{2} V_{3}=500 \mathrm{~cm}^{-1}, V_{6}=0$, $\zeta^{\prime \prime \prime}=\frac{1}{2} \zeta, \zeta^{\prime}=0.95 \zeta, \zeta^{\prime \prime}=0.95 \zeta^{\prime}$. If we take $\zeta=2249 \mathrm{~cm}^{-1}, X=-1620 \mathrm{~cm}^{-1}, Y=14670 \mathrm{~cm}^{-1}$, $Z=22400 \mathrm{~cm}^{-1}$ we find the following energy level positions:

\begin{tabular}{lr}
\hline \hline & $\mathrm{cm}^{-1}$ \\
$E(3-)$ & 0 \\
$E(2-)$ & 306 \\
$E(2+)$ & 6754 \\
$E(3+)$ & 8162 \\
$E(1-)$ & 18108 \\
$E(1+)$ & 21091
\end{tabular}

The conclusion we draw from these calculations is that for moderate values of the crystalline fields and various amounts of covalence the spin-orbit coupling constant and the parameters of the ligand field are fairly well determined. $\zeta$ probably lies in the range 2070 to $2250 \mathrm{~cm}^{-1}, X$ lies between -600 and $-1720 \mathrm{~cm}^{-1}$, and $Y$ lies between 14500 and $15500 \mathrm{~cm}^{-1}$.

The effect of the nonaxial field $V_{6}$ is to raise the $\mid 2->$ level relative to $\mid 3->$. This perturbation is roughly quadratic in $V_{6}$. The perturbations by $V_{3}$ and $V_{3}^{\prime}$ (also quadratic) can move the $\mid 2->$ level either way relative to $\mid 3->$. The effect of reductions in $\zeta^{\prime}$ and $\zeta^{\prime \prime}$ is to raise the $\mid 2->$ level. It is therefore likely that this level is higher than $200 \mathrm{~cm}^{-1}$, but it is probably lower than $1400 \mathrm{~cm}^{-1}$.

\section{Spectroscopic Splitting Factors}

In I we gave approximate formulas for the $g$-values and the susceptibility of the neptunyl ion. More complete formulas will be given here.

It follows from the interaction matrices that there are three types of states for the neptunyl ion. They are

$$
|a>=|-3+><-3+|a>+|-2-><-2-|a>+| 3+><3+|a\rangle
$$

$$
+|1-><1-| a>+|0+><0+| a>,
$$




$$
\begin{aligned}
& |b>=| 3-><3-|b>+| 2+><2+|b>+|-3-><-3-\mid b> \\
& +|-1+><-1+| b>+|0-><0-| b>, \\
& |c>=| 2-><2-|c>+|-2+><-2+|c>+| 1+><1+|c\rangle
\end{aligned}
$$

$$
+|-1-><-1-| c>
$$

The ground state is a Kramers doublet of types $|a\rangle, \mid b>$.

In I we defined our basic states $|a\rangle$ and $|b\rangle$ the other way round (i.e., $\mid 3->$ and $\mid-3+>$ respectively in first approximation) which has the disadvantage that $g_{y y}$ is of opposite sign to $g_{x x}$, so that the axial symmetry of the magnetic properties is lost in the formulation. Our present choice of basic states is consistent with magnetic axial symmetry.

The coefficients occurring in $|b\rangle$ are equal in magnitude to those in $|a\rangle$. From Kramers' theorem one has

$$
\begin{aligned}
& <0-|b>=<0+| a>^{*} \\
& <3-|b>=-<-3+| a>^{*} \\
& <2+|b>=-<-2-| a>^{*} \\
& <-3-|b>=-<3+| a>^{*} \\
& <-1+|b>=<1-| a>^{*}
\end{aligned}
$$

In the double nitrates isomorphous with $\mathrm{Rb}\left(\mathrm{UO}_{2}\right)\left(\mathrm{NO}_{3}\right)_{3}$ the symmetry of the crystalline field is such that if the $c$-axis is chosen as $z$-axis, and an axis joining the center of a nitrate group to the neptunium atom is chosen as $y$-axis, all matrix elements $V_{6}, V_{3}, V_{3}^{\prime}$ are real, and the coefficients above are all real.

In calculating the matrix elements of the magnetic moment operator it is necessary to allow for possible $l$-reduction $[8,9]$. The $m_{l}= \pm 1$ orbitals may be partially used in $\pi$-bonding as already described. We shall denote the appropriately reduced value of $L$ by $k_{1} L$. Similarly, the $m_{l}= \pm 2$, \pm 3 orbitals may overlap the oxygen atoms which form the equatorial ring. We shall denote the reduced $L$ 's by $k_{2} L$ and $k_{3} L$, respectively. (We shall assume that matrix elements of $\mathbf{L}$ between the states with $m_{l}= \pm 2$ and \pm 3 are reduced by $\sqrt{k_{2} k_{3}}$, etc.). One then finds the following matrix elements of the magnetic moment operator:

$$
\begin{gathered}
<a^{\prime}|\mathbf{L}+2 \mathbf{S}| a>=\left\{\left(1-3 k_{3}\right)<a^{\prime}|-3+><-3+| a>-\left(2 k_{2}+1\right)<a^{\prime}|-2-><-2-| a>\right. \\
\left.+\left(3 k_{3}+1\right)<a^{\prime}|3+><3+| a>+\left(k_{1}-1\right)<a^{\prime}|1-><1-| a>+<a^{\prime}|0+><0+| a>\right\} \mathbf{k} \\
<b|\mathbf{L}+2 \mathbf{S}| a>=\left\{\frac{1}{2} \sqrt{6 k_{3} k_{2}}[<b|2+><3+| a>+<b|-3-><-2-| a>]\right. \\
+<b|3-><3+| a>+<b|-3-><-3+| a> \\
+\sqrt{3 k_{1} k_{0}}[<b|0-><1-| a>+<b|-1+><0+| a>] \\
+<b|0-><0+| a>\}(\mathbf{i}+i \mathbf{j})
\end{gathered}
$$


$<c|\mathbf{L}+2 \mathbf{S}| a>=\{<c|-2+><-2-| a>+<c|1+><1-| a>$

$$
\begin{gathered}
\quad+\frac{1}{2} \sqrt{6 k_{3} k_{2}}<c|-2+><-3+| a> \\
+\frac{1}{2} \sqrt{10 k_{2} k_{1}}[<c|2-><1-| a>+<c|-1-><-2-| a>] \\
\left.\quad+\sqrt{3 k_{1} k_{0}}<c|1+><0+| a>\right\}(\mathbf{i}-i \mathbf{j})
\end{gathered}
$$

It follows that

$$
\begin{aligned}
& g_{||}=2\left\{\left(1-3 k_{3}\right)|<-3+| a>\left.\right|^{2}-\left(2 k_{2}+1\right)|<-2-| a>\left.\right|^{2}+\left(3 k_{3}+1\right)|<3+| a>\left.\right|^{2}\right. \\
& \left.+\left(k_{1}-1\right)|<1-| a>\left.\right|^{2}+|<0+| a>\left.\right|^{2}\right\} \\
& g_{\perp}=2\left\{-\sqrt{6 k_{3} k_{2}}<3+|a><-2-| a>-2<3+|a><-3+| a>\right. \\
& \left.+2 \sqrt{3 k_{1} k_{0}}<0+|a><1-| a>+<0+\mid a>^{2}\right\} .
\end{aligned}
$$

If we neglect nonaxial fields, $\mid a>$ contains only $\mid-3+>$ and $\mid-2->$, and $\mid b>$ only $\mid 3->$ and $\mid 2+>$, from which it is easily seen that $g_{\perp}$ is zero. In fact if we take the parameters $\zeta=2248$ $\mathrm{cm}^{-1}, X=-1716 \mathrm{~cm}^{-1}, V_{3}=V_{3}^{\prime}=V_{6}=0$ we find

$$
\begin{aligned}
& |a>=0.8883|-3+>-0.4593 \mid-2-> \\
& |b>=-0.8883| 3->+0.4593 \mid 2+>
\end{aligned}
$$

and

$$
\begin{aligned}
& g_{\|}=-\left\{0.7891\left(6 k_{3}-2\right)+0.2109\left(4 k_{2}+2\right)\right\} \\
& g_{\perp}=0 .
\end{aligned}
$$

With $k_{3}=k_{2}=1$, one obtains $g_{\|}=-4.4218$. With $k_{3}=k_{2}=0.9, g_{\|}=-3.8640$.

The experimental values for rubidium neptunyl nitrate $[5,10]$ are $\left|g_{\|}\right|=3.405 \pm 0.008$ and $\left|g_{\perp}\right|=0.205 \pm 0.006$. They differ from the above values in that, first $g_{\perp} \neq 0$, and secondly $\left|g_{\|}\right|$is lower. As was discussed in $\mathrm{I}$, the effect of $V_{6}$ is to give rise to a nonzero $g_{\perp}$ and to some reduction in $\left|g_{\|}\right|$. Orbital reduction will further reduce $\left|g_{\|}\right|$.

$V_{3}$ and $V_{3}^{\prime}$ will also modify the $g$-values slightly, but their contributions are secondary so that if we wish to determine the parameters of the theory approximately we can concentrate on $V_{6}, k_{3}$, and $k_{2}$. We shall assume that $k_{3}=k_{2}$ for simplicity. If we set

$$
\zeta=2175 \mathrm{~cm}^{-1}, X=-1220 \mathrm{~cm}^{-1}, V_{6}=1000 \mathrm{~cm}^{-1}, V_{3}=V_{3}^{\prime}=0,
$$

which parameters give energy levels in the correct places, we have

$$
\begin{aligned}
& |a>=0.8975|-3+>-0.4262|-2->-0.1134| 3+> \\
& |b>=-0.8975| 3->+0.4262|2+>+0.1134|-3->.
\end{aligned}
$$

For $k_{3}=k_{2}=1$, one has $g_{\mid]}=-4.2084, g_{\perp}=0.1706$. With $k_{3}=k_{2}=0.9$, this becomes modified to $g_{\|}=-3.6602, g_{\perp}=0.1942$. With $k_{3}=k_{2}=0.85$ the values are $g_{\|}=-3.3861$ and $g_{\perp}=0.2060$. 
These results indicate that $V_{6}$ around $1000 \mathrm{~cm}^{-1}$, and orbital reduction to about 85 percent are adequate hypotheses to account for the resonance results. Such a large amount of orbital reduction implies considerable overlap of the $5 f$ wave functions on the oxygen atoms of the nitrate groups. Such partial covalency in the formation of a hexanitrato-uranyl ion has already been considered on chemical grounds by Coulson and Lester [11]. If this deduction is correct, the parameter $\zeta$ which enters into our calculations does not have the value of the spin-orbit coupling constant in the free neptunyl ion, but a value reduced by about 0.85 . It follows that in the free ion $\zeta=2178 / 0.85$ $=2560 \mathrm{~cm}^{-1}$. Too much should not be made of this estimate, however, since we are combining optical data from aqueous solution with magnetic data from $\mathrm{Rb}\left(\mathrm{NpO}_{2}\right)\left(\mathrm{NO}_{3}\right)_{3}$, and the "crystalline" fields may be quite different in the two systems. However, such a line of thought suggests that the apparent discrepancy between the values of $\zeta$ derived from the $\mathrm{NpO}_{2}^{++}$solution absorption spectrum and from the $\mathrm{NpF}_{6}$ spectrum (about $2400 \mathrm{~cm}^{-1}$ ) is capable of resolution.

In future work on neptunyl compounds it would be worthwhile to study the Zeeman effect in the spectra of crystals, since the Zeeman splittings furnish a valuable test of the identification of the lines. One expects large Zeeman splittings for several of the levels when the magnetic field is parallel to the ionic axis.

We therefore give, as a guide, the approximate values of the spectroscopic splitting factors deduced from simple calculations (which neglect orbital reduction).

\begin{tabular}{r|r|r}
\hline \hline State & Energy & $\begin{array}{c}\left|g_{\|}\right| \text {(uncorrected for } \\
\text { orbital reduction) }\end{array}$ \\
\hline & $c^{-1}$ & \\
$\mid-3+>$ & 0 & 4.2 \\
$2->$ & 664 & 2.1 \\
$2+>$ & 6752 & 4.3 \\
$3+>$ & 8168 & 5.6 \\
$1->$ & 18100 & 0.0 \\
$1+>$ & 21100 & 3.9 \\
\hline
\end{tabular}

The absorption spectrum of crystals of $\mathrm{Rb}\left(\mathrm{NpO}_{2}\right)\left(\mathrm{NO}_{3}\right)_{3}$ (or mixed crystals with the isomorphous uranyl salt) will probably give sharp enough lines at low temperatures to permit the Zeeman structure to be resolved. Each line will be split into four components, two of which will be strong and two weak, because of the operation of the selection rule $\Delta m_{s}=0$. The approximate splittings to be expected for the vibrationless $(0-0)$ electronic transitions are as follows:

\begin{tabular}{c|c|c}
\hline \hline & \multicolumn{2}{|l}{} \\
\multirow{2}{*}{ Transition } & Expected Zeeman splitting/“Normal” Zeeman splitting \\
\cline { 2 - 3 } & Strong components & Weak components \\
& & \\
$|-3+>\rightarrow|-2+>$ & 2.1 & 6.3 \\
$|-3+>\rightarrow| 2+>$ & 8.5 & 0.1 \\
$|-3+>\rightarrow| 3+>$ & 9.8 & 1.4 \\
$|-3+>\rightarrow|-1+>$ & 4.2 & 4.2 \\
$|-3+>\rightarrow| 1+>$ & 8.1 & 0.3 \\
\hline
\end{tabular}


These are large Zeeman separations, and should not be difficult to detect provided the absorption lines in the crystalline state are sharp. In view of the sharpness of the lines in the absorption spectrum of $\mathrm{RbUO}_{2}\left(\mathrm{NO}_{3}\right)_{3}$, where a considerably smaller Zeeman effect was detected [12], there is reason to believe that this condition will be satisfied.

\section{Nuclear Hyperfine Structure}

The nuclear hyperfine structure is described by the term

$$
A S_{z}^{\prime} I_{z}+B\left(S_{x}^{\prime} I_{x}+S_{y}^{\prime} I_{y}\right)+P I_{z}^{2}
$$

in the spin Hamiltonian, where $S^{\prime}$ is the fictitious spin. The derivation of the hyperfine structure parameters $A, B$, and $P$ from the characteristics of the states $|a\rangle$ and $|b\rangle$ was described in $\mathrm{I}$, and can be deduced from equations (2.3), (2.8), and (2.9) of Abragam and Pryce [13]. We here wish to add two points to the previous discussion.

First, consequent on the interchange of the basic states $|a\rangle$ and $|b\rangle$ as compared with I, the sign of $A$ (like that of $\left.g_{\|}\right)$must be reversed. This reversal is not trivial, for the resonance data, while not determining the sign of $A$, do determine the relative signs of $A$ and $P$. Theoretical prediction of the sign of $A$ therefore implies theoretical prediction of the sign of the quadrupole moment of the nucleus, and our previous deduction was wrong. The present interpretation of the data of Bleaney et al. [5] and Llewellyn (quoted by Bowers and Owen [10]) for $\mathrm{Np} p^{237}$ in $\mathrm{Rb}\left(\mathrm{NpO}_{2}\right)\left(\mathrm{NO}_{3}\right)_{3}$ is

$$
\begin{aligned}
A & =-0.16547 \pm 0.00008 \mathrm{~cm}^{-1} \\
|B| & =0.01782 \pm 0.00003 \mathrm{~cm}^{-1} \\
P & =+0.03017 \pm 0.00007 \mathrm{~cm}^{-1} .
\end{aligned}
$$

The $\operatorname{sign}$ of $A$ is based on the assumption that the nuclear moment is positive. According to (5.9) of $\mathrm{I}$, the sign of the nuclear quadrupole moment is opposite to that of $P$, and is thus negative.

The magnetic hyperfine coupling arises in part from the magnetic field of the $5 f$ electron, and in part from small admixtures of $s$-states, which are due to perturbations such as configuration interaction. In the present case, there is a perturbation resulting in $s$-electron coupling, which we omitted in I, namely the $V_{3}^{3}$ term in the crystalline potential, which couples the $5 f, m_{l}= \pm 3$ states to $s$-states ( $7 s$ antibonding or $8 s$, etc.). This coupling leads to an additional contribution to the expressions (5.6) of I for $A$ and $B$.

Assuming, for illustration, that the ground state is given by

$$
|a>=0.8975|-3+>-0.4262|-2->-0.1134| 3+>\text {, etc. }
$$

we obtain

$$
\begin{aligned}
& A=-\left(13.305+1.273 \kappa-\kappa^{\prime}\right) \Upsilon \beta \beta_{N}<r^{-3}> \\
& B=-\left(0.2589+0.4071 \kappa-\kappa^{\prime}\right) \Upsilon \beta \beta_{n}<r^{-3}>
\end{aligned}
$$

where $\kappa$ is the usual parameter characterizing $s$-electron admixture (through configuration interaction) and $\kappa^{\prime}$ is a parameter describing the admixture through $V_{3}^{3} . \quad \kappa^{\prime}$ is probably small for it is quadratic in the admixture, unlike $\kappa$, which is linear [14].

Nothing is known about the magnitude of $\kappa$, but it seems unlikely to be appreciable. We may therefore make an estimate of the nuclear moment $\mu_{N}$ from the experimental value of $A$ by neglecting the $\kappa$ and $\kappa^{\prime}$ terms. It is necessary to know $\left\langle r^{-3}\right\rangle$. 
Both $\zeta$ and $\left\langle r^{-3}\right\rangle$ were calculated by Foglio and Pryce [15] on the basis of a Thomas-Fermi model. They concluded that this model leads to values of $\zeta$ and $\left\langle r^{-3}\right\rangle$ which are too low, but that the calculated ratio $\zeta /\left\langle r^{-3}\right\rangle$ is fairly accurate. If we take $\left.\zeta /<r^{-3}\right\rangle \sim 360 \mathrm{~cm}^{-1} /$ atomic unit as indicated by their results and $\zeta \sim 2160 \mathrm{~cm}^{-1}$ as indicated by the previous discussion in this paper we find $\left\langle r^{-3}\right\rangle \sim 6.0$ atomic units $\left(40 \times 10^{24} \mathrm{~cm}^{-3}\right)$

We then obtain

$$
\begin{aligned}
\mu_{N} & =\Upsilon I=\frac{5}{2} \frac{0.16547}{6.0 \times 1.5913 \times 10^{-3} \times 13.305} \\
& =3.2 \text { nuclear magnetons. }
\end{aligned}
$$

There are at least three sources of error here, apart from the experimental error in the determination of $A$ : - error in $\left\langle r^{-3}\right\rangle$, which we set at \pm 20 percent; error from neglecting $\kappa$ and $\kappa^{\prime}$, which we set at \pm 10 percent; and error from (somewhat arbitrarily) using the ground state above, which however is negligible in comparison with the other two. We may therefore write

$$
\mu_{N}=3.2+0.9 \text { n.m. }
$$

\section{Magnetic Susceptibility}

The susceptibility can be written in the form

$$
\chi=\frac{N \beta^{2}}{12 k}\left(g_{\|}^{2}+2 g_{\perp}^{2}\right)+a
$$

here $k$ is the Boltzmann constant), provided the temperature is sufficiently low for the $\mid 2->$ level to be effectively unpopulated. We wish here to estimate the value of $a$, which is given by

$$
a=\chi_{\mathrm{core}}+\frac{2}{3} N \beta^{2} \Sigma_{i} \frac{|<0| \mathbf{L}+2 \mathbf{S}|i>|^{2}}{\left(E_{i}-E_{0}\right)}
$$

The contribution of the core, $\chi_{\text {core }}$, we have previously estimated to be $57 \times 10^{-6} \mathrm{emu} / \mathrm{mole}$.

The dominant term in this sum arises from the $\mid 2->$ level, because it is so low. With the parameters we have chosen for the ground state, and $k_{3}=k_{2}=0.85$ we obtain for this dominant term

$$
\frac{0.1248}{\triangle E\left(\mathrm{~cm}^{-1}\right)} \mathrm{emu} / \mathrm{mole}
$$

The contributions from the remaining leveis total about $25 \times 10^{-6}$. We therefore estimate

$$
a \sim\left(82+\frac{0.1248}{\triangle E}\right) \times 10^{-6} \mathrm{emu} / \mathrm{mole} .
$$

In $\mathrm{Na} \mathrm{NpO} \mathrm{N}_{2}\left(\mathrm{CH}_{3} \mathrm{COO}\right)_{3}$ the observed [6] value of $a$ is $194 \times 10^{-6}$. The calculated value of $a$ would also be $194 \times 10^{-6}$ if the $\mid 2->$ level were at $1100 \mathrm{~cm}^{-1}$. This position for the $\mid 2->$ level is within the limits suggested by the calculations in section 4 .

\section{Conclusion}

In this paper we have discussed the magnetic and spectroscopic properties of the neptunyl ion, and have presented an interpretation of these properties which is based on some hypotheses concerning the structure of the ion and its environment. The hypotheses seem to be fully justified a priori, by crystal structure data on uranyl and neptunyl compounds, and a posteriori, by detailed agreement between calculated and observed quantities. 
We have dealt first with the solution absorption spectrum, and have shown how the spin-orbit coupling constant and the parameters of the ligand and crystal fields can be determined from the positions of the absorption peaks. We have pointed out an important ambiguity in the interpretation of the spectrum and have tried to indicate that there is uncertainty concerning the precise values of the parameters. The ambiguity and uncertainty stem partly from the complexity of the physical system involved and partly from the limited extent and accuracy of the experimental data. We have tried to treat, in a quantitative way, the effect of covalence on the positions of the energy levels.

The available magnetic data come from electron spin resonance experiments and from susceptibility measurements. We have shown that theoretical and experimental values for the spectroscopic splitting factors agree if a crystal field of appropriate symmetry and magnitude is present, and if covalent effects which effectively reduce the orbital angular momentum are also present. The same assumptions which lead to agreement of calculated and experimental values for the spectroscopic splitting factors lead also to agreement of calculated and experimental values for the susceptibility.

We believe the principal factors which determine the magnetic and spectroscopic properties of uranyl type ions are now known. Ligand field, crystal field, and covalent effects make the behavior of these ions rather complicated.

A second paper on the properties of the $\left(\mathrm{PuO}_{2}\right)^{++}$and the $\mathrm{NpO}_{2}^{+}$ions is being prepared. A more convincing interpretation than heretofore of the rather complex absorption spectra of these ions can now be given. Eventually a long deferred paper on the americyl and $\mathrm{PuO}_{2}^{+}$ions may follow.

We thank Clyde A. Hutchison, Jr., for many stimulating and enlightening discussions, and M. Fred for information and discussions concerning the absorption spectra.

\section{Appendix}

Plots oi absorption spectra are usually given in terms of molecular extinction coefficient or absorbance as a function of wavelength. The molecular extinction coefficient $\epsilon$ is defined as follows:

$$
\epsilon=\frac{-\log _{10}(\text { transmittancy })}{\text { Molarity } \times \text { length of cell (in } \mathrm{cm})} \text {. }
$$

The transmittancy is defined as the ratio $I / I_{0}$ where $I$ is the radiant flux transmitted and $I_{0}$ is the radiant flux incident. The molarity is the number of gram molecular weights of the absorbing substance per liter of solution. The absorptance is [1-transmittancy] and the absorbance is $\log _{10}$ (absorptance).

Another term in common use is the specific extinction, $k$. This is defined as

$$
k=E / c d
$$

where

$$
\begin{aligned}
& E=\text { extinction }=\log _{10} I_{0} / I \\
& c=\text { concentration in grams/liter } \\
& d=\text { thickness of cell in } \mathrm{cm} .
\end{aligned}
$$

Evidently $\epsilon=$ molecular weight $\times k$. 
The energy absorbed per unit time at energy $h v$ is $N w h \nu$ where $N$ is the number of absorbers and $w$ is the probability that a transition will take place. The energy absorbed per unit time at energy $h \nu$ per volume $V$ is $N w h \nu / V$. For an oscillating magnetic field $\tilde{H} \cos \omega t$ the incident energy per $\mathrm{cm}^{2}$ per sec is $c \tilde{H}^{2} / 8 \pi$. Hence

$$
\frac{\frac{\text { Energy absorbed }}{\text { volume second }}}{\frac{\text { energy incident }}{\mathrm{cm}^{2} \text { second }}}=\frac{\frac{w h \nu N}{V}}{\frac{c \tilde{H}^{2}}{8 \pi}}=-\frac{d I}{I d y}=\alpha \text {, say }
$$

where

$$
I=I_{0} e^{-\alpha y}
$$

or

$$
\log _{e} I / I_{0}=-\alpha y \text {. }
$$

Let us put these relations into conformity with the previous notation. We use $d$ instead of $y$. Then

$$
2.303 \log _{10} I / I_{0}=-\alpha d \text {. }
$$

But

$$
\begin{aligned}
-\log _{10} I / I_{0}=E & =k \times \text { concentration } \times d \\
& =\epsilon \times \text { concentration } \times d / M,
\end{aligned}
$$

$M$ being the molecular weight. Hence

and

$$
\begin{aligned}
& \alpha=2.303 \epsilon \times \text { concentration } / M \\
& \epsilon=\frac{M}{2.303 \times \text { concentration }} \frac{w h \nu N / V}{c \tilde{H}^{2} / 8 \pi} .
\end{aligned}
$$

For magnetic dipole absorption we have

$$
w=\frac{2 \pi}{\hbar} \beta^{2} \frac{\tilde{H}^{2}}{4} \rho\left(E_{f}\right)
$$

where $\rho\left(E_{f}\right)$ is the density of final states per unit energy range. Hence since

$$
\frac{\text { molecular weight }}{\text { grams/liter }} \frac{\text { number of absorbers }}{\mathrm{cm}^{3}}=\frac{N_{0}}{1000}
$$

we have

$$
\epsilon=\frac{8 \pi^{3} N_{0} \beta^{2} \nu \rho}{2303 c}\left(E_{f}\right)
$$

$N_{0}$ is the Avogadro number.

As a measure of the strength of an absorption line we shall take

$$
k \int \epsilon \frac{d \nu}{\nu}
$$


where $k$ is a constant chosen to make the strength corresponding to a magnetic dipole transition equal to one. In terms of our previous formula we must have

$$
k \int \frac{8 \pi^{3} N_{0} \beta^{2}}{2303} \frac{\nu}{c} \rho\left(E_{f}\right) \frac{d \nu}{\nu}=1
$$

or

$$
k=\frac{2303 h c}{8 \pi^{3} \beta^{2} N_{0} \int \rho\left(E_{f}\right) d E_{f}}
$$

Since only one final state is accessible to a magnetic dipole we have

$$
k=\frac{2303 h c}{8 \pi^{3} \beta^{2} N_{0}}=35.587
$$

In practice it is sometimes inconvenient to calculate $\int \epsilon d \nu / \nu$ so we make the approximation that the absorption peaks have a Gaussian frequency distribution. It then follows that the area under the peaks is approximately

$$
\int \frac{\epsilon d \nu}{\nu}=1.064 \frac{\epsilon_{\max } \Delta \nu}{\nu_{0}}
$$

where $\Delta \nu$ is the width of the peak at half intensity, and $\epsilon_{\max }$ is the molecular extinction coefficient at the frequency $\nu_{0}$ where the absorption is maximal. The strength of a Gaussian peak therefore is $37.9 \epsilon_{\max } \Delta \nu / \nu_{0}$.

Since we make considerable use of Waggener's spectroscopic measurements it is worth noting that he plots a quantity he calls "absorbance" versus wavelength. This quantity is in reality the extinction $\log _{10} I_{0} / I$.

\section{References}

[1] J. C. Eisenstein and M. H. L. Pryce, Proc. Roy. Soc. A229, 20 (1955) and 238, 31 (1956).

[2] J. C. Hindman, private communication.

[3] W. C. Waggener, J. Phys. Chem. 62, 382 (1958).

[4] W. H. Zachariasen and H. A. Plettinger, Acta Cryst. 12, 526 (1959).

[5] B. Bleaney, P. M. Llewellyn, M. H. L. Pryce, and G. R. Hall, Phil. Mag. 45, 992 (1954).

[6] D. M. Gruen and C. A. Hutchison, J. Chem. Phys. 22, 386 (1954).

[7] J. C. Eisenstein and M. H. L. Pryce, Proc. Roy. Soc. A255, 181 (1960).

[8] K. W. H. Stevens, Proc. Roy. Soc. A2 19, 542 (1953).

[9] J. Owen, Proc. Roy. Soc. A22 7, 183 (1955).

[10] K. D. Bowers and J. Owen, Rep. Progr. Phys. 18, 304 (1955).

[11] C. A. Coulson and G. R. Lester, J. Chem. Soc. 1956, 3650.

[12] G. H. Dieke and A. B. F. Duncan, Spectroscopic Properties of Uranium Compounds (National Nuclear Energy Series III, 2), New York (McGraw-Hill Book Co., New York, N.Y., 1949).

[13] A. Abragam and M. H. L. Pryce, Proc. Roy. Soc. A205, 135 (1951).

[14] A. Abragam, J. Horowitz and M. H. L. Pryce, Proc. Roy. Soc. A230, 169 (1955).

[15] M. E. Foglio and M. H. L. Pryce, Mol. Phys. 4, 287 (1961).

(Paper 69A3-341) 